\title{
Psychopathology Associated With Sexual Abuse: The Importance of Complementary Designs and Common Ground
}

\author{
Michael R. Nash \\ University of Tennessee
}

Timothy L. Hulsey

Southwest Texas State University

\author{
Robert A. Neimeyer \\ University of Memphis
}

Warren Lambert

Vanderbilt University

\begin{abstract}
In their recent longitudinal study of youth victimization and consequent traumatization, S. BoneyMcCoy and D. Finkelhor (1996) contrast their position with previous retrospective research (S. Harter, P. Alexander, \& R. A. Neimeyer, 1988; M. R. Nash, T. C. Hulsey, M. C. Sexton, T. L. Harralson, \& W. Lambert, 1993a), arguing that their data support the impact of victimization per se, independent of the moderating effect of family environment. Because Boney-McCoy and Finkelhor's argument may misrepresent the results of such studies, this article (a) clarifies the actual findings of previous retrospective studies of abuse, (b) suggests methodological limitations both in BoneyMcCoy and Finkelhor's research and in that of S. Harter et al., 1998, and M. R. Nash et al., 1993a, that should be remedied by future investigators, and (c) argues that both retrospective clinical research and prospective community surveys converge on a common ground, namely, that specific abuse experiences can best be understood and investigated in the context of the prior, contemporaneous, and subsequent family environments in which they occur.
\end{abstract}

Because sexual abuse is typically accompanied by a host of other family problems (e.g., distorted family relationships, secrecy, enmeshment, violence, poverty, and substance abuse), it is difficult to track the impact of abuse per se separate from the context in which it occurs. In fact, most researchers have moved away from the magic bullet idea that a specific sexual event invariably leads to a specific outcome or syndrome, instead emphasizing that the psychological effects of abuse must be understood (and studied) within the context of the family-social environment in which the abuse is embedded (e.g. Briere \& Elliot, 1993; Browne \& Finkelhor, 1986). Nonetheless, investigators differ in their views concerning the relative contributions of abuse per se, as opposed to the family environment that permits abuse, in determining the psychological sequelae of the experience.

In a recent study, Boney-McCoy and Finkelhor (1996) highlighted this difference and contrasted their work with our own (Harter, Alexander, \& Neimeyer, 1988; Nash, Hulsey, Sexton, Harralson, \& Lambert, 1993a), arguing that retrospective research designs like those in our studies are inherently limited in their ability to determine the relative impact of abuse events versus broader family variables on subsequent adjustment. Of course, well-conducted retrospective studies with women or

Michael R. Nash, Department of Psychology, University of Tennessee; Robert A. Neimeyer, Department of Psychology, University of Memphis; Timothy L. Hulsey, Department of Psychology, Southwest Texas State University; Warren Lambert, Department of Psychology, Vanderbilt University.

Correspondence concerning this article should be addressed to Michael R. Nash, Department of Psychology, University of Tennessee, 307 Austin Peay Building, Knoxville, Tennessee 37996-0900. children who report past abuse can help to identify possible contextual mediators between the event of abuse and subsequent psychological symptoms. However, such studies cannot adequately assess cause, a point we acknowledged (Nash, Hulsey, Sexton, \& Harralson, \& Lambert, 1993b).

It is a hopeful sign, then, that in their recent article on victimization and psychopathology, Boney-McCoy and Finkelhor (1996) used a quasi-experimental prospective design attempting to weigh the relative pathogenic properties of sexual abuse per se and of social--family context. In a large random-sample telephone survey, they interviewed 1,433 children $10-16$ years old (T1) and then reinterviewed them 15 months later (T2) on matters pertaining to psychological distress, family relationships, and victimization. They concluded that their study advances the idea that there is a causal link between victimization and subsequent psychological distress (Boney-McCoy \& Finkelhor, 1996, p. 1414) and further concluded that victimization makes a contribution to symptomatology that is independent of social-family context (1996, p. 1415). The prospective design of this ambitious study is a welcome feature that partially compensates for some of its other weaknesses. However, because they quoted and cited work carried out in our independent laboratories (Harter et al., 1988; Nash et al., 1993a) and because they persistently contrasted their finding with ours, we want to jointly communicate the concerns we have about (a) the adequacy of their sample and research design in supporting the inferences they have drawn and (b) the misrepresentation of our position and our findings concerning the complicated problem of event and context in the genesis of psychopathology. In doing so, we hope to reinforce the necessity of recognizing the strengths and limitations of complementary designs and also to define a common ground that will provide greater direction to 
the work of future investigators in the challenging area of sexual abuse.

\section{Complementary Samples}

Although a focus on various forms of abuse is in itself legitimate, the abuse experiences sampled by Boney-McCoy and Finkelhor (1996) limit their ability to address the hypothesis that family environment accounts for much of the impact of such abuse. This is because only 5 of the 93 sexually abused children in the Boney-McCoy and Finkelhor study were abused by a family member or surrogate (see their Table 2, p. 1411), whereas all participants in one of our own studies (Harter et al., 1988) and 58\% in the other (Nash et al., 1993a) were victims of in-family abuse. Logically, one would not expect that home environment would play the same role in explaining, mitigating, or exacerbating symptomatolgy if the abuser were not part of that home environment. Put more strongly, it is quite difficult to even test the hypothesis that the effect of intrafamily abuse is associated with family environment if one virtually excludes intrafamily victimization. It is precisely in the case of intrafamilial abuse that we would expect family context to have its greatest effect, an effect that was indeed more clearly evidenced in our studies.

\section{Complementary Methods}

Of course, no single study-retrospective or prospective, community-based or clinical-can definitively answer the questions researchers have about psychopathology and abuse. As all investigators in this area plan their studies, they face special challenges regarding measurement and design that affect what can be said in the Discussion section. These special measurement and design considerations include participant reactivity, stability-relevance of outcome and predictor variables, timing of measurement, and indexing of change.

\section{Response Format}

Boney-McCoy and Finkelhor (1996) rightly acknowledged that in response to their anonymous telephone survey (conducted in the home) children might indeed conceal in-family victimization (because they were using the family phone and speaking out loud). By virtue of their reactivity to this measurement procedure, children might also be disinclined to reveal other unpleasant aspects of the family environment, as well as their psychological status, thus further reducing the likelihood of detecting a link between general family dysfunction and the impact of abuse per se.

\section{The Measurement of Family Environment}

Our approach to measuring family context (which produced stronger relationships between family factors and pathology) was to rely on widely used and reasonably well-validated measures of family adaptability, cohesion and social adjustment (Bloom, 1985; Olson, Russell, \& Sprenkle, 1983), whereas Boney-McCoy and Finkelhor constructed a brief, as yet unvalidated seven-item questionnaire of family interaction. Although a pilot measure of this kind may nonetheless yield useful data, we wonder whether the content of their scale adequately addresses the most relevant family relationships. Specifically, four of the seven questions on the scale focus on the adult in the household who "know [s] most about [their] activities" (Boney-McCoy \& Finkelhor, 1996, p. 1408), rather than on both parents, at minimum, or on the family environment, more generally. The preponderance of questions, then, may have implicitly excluded the child's perceived relationship with the abusing parent and, more commonly, with the parent currently out of favor. If future investigators want to test the effect of family environment on mental health outcomes, then they may be better served by using measures having demonstrated reliability and validity. A potentially invalid measure or a lack of focus on those persons or relationships likely to contribute significantly to the child's distress or well-being would clearly inhibit detection of relationships between family environment and abuse outcome. Under these circumstances, it is not surprising that Boney-McCoy and Finkelhor's (1996) questionnaire failed to account for more of the variance in outcome. They make this point when they noted, "A more comprehensive family environment measure might have reduced the victimizationsymptomatology association further' (1996, p. 1415).

\section{Temporal Focus of Assessment}

Abuse takes place in a temporal context of previous, contemporaneous, and future family interactions. Theoretically, any of these past, present, or future aspects of the family environment could moderate the impact of an abuse episode. In Boney-McCoy and Finkelhor's (1996) case, the study's central concern is with the role of previous family functioning in accounting for subsequent distress associated with abuse and other forms of victimization. In contrast, the Harter et al. (1988) study suggests that much (although not all) of the impact of sexual abuse can be accounted for by the current family environment in which the abuse takes place (an environment likely marked by power assertion, blurred generational boundaries, social isolation, and enmeshment). Whereas both perspectives are legitimate, both are also partial, and we encourage future researchers to study the effects of family environment before, during, and after the time of abuse.

\section{Measurement of Outcome}

Boney-McCoy and Finkelhor's (1996) indexing of psychological distress (depression and "PTSD-related symptoms," p. 1408 ) was conducted with broadly adequate measures. Nonetheless, it is important to recognize that they used two quite different measures of PTSD-related symptoms at T1 and T2, potentially undermining their attempted control for prior symptomatology. Although Boney-McCoy and Finkelhor (1996) suggested that the correlation of .40 between these scales justifies using them as repeated measures, it surely does not inspire confidence that these two scales are indexing the same thing. Thus, we suggest that future investigators adopt more stringent controls for prior symptomatology if their goal is to isolate the effect of abuse and family environment on mental health outcome. 


\section{An Appeal for Common Ground}

After all is said and done, how do the results of BoneyMcCoy and Finkelhor's studies compare with the results of our own retrospective studies? Boney-McCoy and Finkelhor have consistently pressed home the point that their findings and conclusions are at odds with ours; for example, they stated that "several of these studies suggest that the effects of victimization are 'canceled out' by the inclusion of family relationship measures . . " (1996, p. 1415). This is not what we stated, nor is it what we intended to suggest. In another particularly vexing example, Boney-McCoy and Finkelhor selectively quoted a portion of the final sentence in the Nash et al. (1993a) work as follows (and we use the exact punctuation, quotation marks and wording as they appear in the Boney-McCoy and Finkelhor article):

On the basis of such analyses, Nash et al. concluded that "much of the adult pathology observed to be associated with childhood sexual abuse may be a product of a generally pathogenic family environment (1993, p. 282)." (Boney-McCoy \& Finkelhor, 1996, p. 1407)

Below is the actual wording of the entire Nash et al. (1993a) sentence. The portion extracted by Boney-McCoy and Finkelhor is underlined:

We contend that, although much of the adult pathology observed to be associated with childhood sexual abuse may be a product of a generally pathogenic family environment, the experience of sexual abuse renders the child especially vulnerable to experiencing her self and her body as fundamentally damaged and painfully inadequate. (Nash et al., 1993a, p. 282)

Although it is important to place the conclusions of previous investigators in their broader context, it is even more important to accurately represent their results. In the Nash et al. (1993a) study these results were clearly articulated. Continuing from the last sentence of the paragraph immediately preceding the one above, Nash et al. noted the following.

. . . even when we controlled for family environment, even when we compared our abused patients with very troubled non-abused patients, we found that women who were sexually abused in childhood must more often contend with a distressing sense that something about them is fundamentally damaged. (Nash et al., 1993a, p. 282)

Thus, Nash et al. found and reported effects for abuse per se, even after controlling for context. Likewise, so did Harter and her colleagues (1988). They found and reported the following.

... subsequent multiple regression analyses also indicated that abuse by a paternal figure and abuse that included intercourse significantly contributed to social maladjustment and to perceived social isolation, respectively, even after the more significant effects of family structure were controlled. (Harter et al., 1988, p. 8).

Both studies then, reported results that acknowledged the deleterious effects of abuse beyond the family context in which it occurred. Our concern is that by creating a tone of polarization in their article, Boney-McCoy and Finkelhor may obscure and inhibit much-needed dialogue on the very complex issue of context and event in the genesis of psychopathology.

What is the disagreement here? Logically there would seem to be two possibilities. First, Boney-McCoy and Finkelhor argue for an effect of victimization independent of context. Do we disagree with them? No. Although we do not believe that their study is a strong test of this position, we do, in fact, concur with the position itself. The importance of event is consistent with our findings; it is consistent with their findings. Thus, both our retrospective research on clinical populations and their surveys of community samples support the notion that event ( sexual victimization in this case) can be a pathogen.

The second possible point of contention might be our message that family-social context probably mediates the effect of victimization, and this must be taken into consideration when inferences are made about the effects of sexual abuse. Do BoneyMcCoy and Finkelhor disagree? No. The importance of context is consistent with their findings (significant independent effect for parent-child relationships on outcome); it is consistent with our findings. Although they may have serious reservations about whether our studies support this point of view, they in fact concur with the basic argument. In their words: ". . . the fact that depression and traumatic stress symptoms can have other antecedents should also be noted by clinicians and policy makers, a finding that may be obscured in cross-sectional research focused exclusively on victimization" (Boney-McCoy \& Finkelhor, 1996, p. 1416). We fully concur.

Is there any real disagreement, then? We believe there is. It is fair to say that we take a very skeptical stance with regard to the relative merit of traumagenic theories of psychopathology that attribute acute and especially long-term impacts of trauma to isolated and objectively defined events without taking into account social and cognitive mediators in the lives of survivors. We think Boney-McCoy and Finkelhor would agree with us to a point, but they may view the relative importance of context differently. However, one imperative about disciplined discourse is that we recognize common ground when there is some. This is all the more important because this area of research is so keenly scrutinized by public and private interests outside the scientific community. We believe there is such common ground and that it is solid. We trust that the message of both longitudinal and cross-sectional research conducted to date is clear: Context and event must be studied together. The best current evidence indicates that both have an effect, although much remains to be learned about their interaction. We hope that clinical investigators will join on this common ground and work to refine both the questions and methods by which we study the impact of traumatic events and pathogenic family environments in the lives of our patients.

\section{References}

Bloom, B. (1985). A factor analysis of self-report measures of family functioning. Family Process, 24, 225-239.

Boney-McCoy, S., \& Finkelhor, D. (1996). Is youth victimization related to trauma and depression after controlling for prior symptoms and family relationships? A longitudinal, prospective study. Journal of Consulting and Clinical Psychology, 64, 1406-1416.

Briere, J., \& Elliott, D. M. (1993). Sexual abuse, family environment, 
and psychological symptoms: On the validity of statistical control. Journal of Consulting and Clinical Psychology, 61, 276-283.

Browne, A., \& Finkelhor, D. (1986). Impact of child sexual abuse: A review of the research. Psychological Bulletin, 99, 66-77.

Harter, S., Alexander, P., \& Neimeyer, R. A. (1988). Long-term effects of incestuous child abuse in college women: Social adjustment, social cognition, and family characteristics. Joumal of Consulting and Clinical Psychology, 56, 5-8.

Nash, M. R., Hulsey, T. C., Sexton, M. C., Harralson, T. L., \& Lambert, W. (1993a). Long-term sequelae of childhood sexual abuse: Perceived family environment, psychopathology, and dissociation. Journal of Consulting and Clinical Psychology, 61, 276-283.
Nash, M. R., Hulsey, T. C., Sexton, M. C., Harralson, T. L., \& Lambert W. (1993b). Reply to comment by Briere and Elliott. Journal of Consulting and Clinical Psychology, 61, 289-290.

Olson, D. H., Russell, C. S., \& Sprenkle, D. H. (1983). Circumplex model of marital and family systems: VI. Theoretical update. Family Process, 22, 69-83.

Received March 17, 1997

Revision received July 15,1997

Accepted July 15, 1997 